\title{
PSFC/JA-00-9
}

\section{Radial Impurity Transport in the H-Mode Transport Barrier Region in Alcator C-Mod}

T. Sunn Pedersen, R.S. Granetz, A.E. Hubbard, I.H. Hutchinson, E.S. Marmar, J.E. Rice, J. Terry

March 2000

\author{
Plasma Science and Fusion Center \\ Massachusetts Institute of Technology \\ Cambridge, MA 02139 USA
}

This work was supported by the U.S. Department of Energy, Cooperative Grant No.

DE-FC02-99ER54512. Reproduction, translation, publication, use and disposal, in whole or in part, by or for the United States government is permitted.

Submitted for publication to Nuclear Fusion. 


\title{
Radial Impurity Transport in the H-Mode Transport Barrier Region in Alcator C-Mod
}

\author{
T. Sunn Pedersen, R.S. Granetz, A.E. Hubbard, \\ I.H. Hutchinson, E.S. Marmar, J.E. Rice, J. Terry \\ MIT Plasma Science and Fusion Center \\ Cambridge, MA 02139, USA
}

\begin{abstract}
Measurements of profiles of soft x-ray emissivity with $1.5 \mathrm{~mm}$ radial resolution are combined with high resolution electron density and temperature measurements in the edge region of the Alcator C-Mod tokamak to facilitate transport analysis of medium-Z impurities during Hmodes. Results from detailed modelling of the radiation and transport of fluorine are compared with the experimental measurements, yielding information about the transport coefficients in the H-mode transport barrier region. We find evidence for a strong inward impurity pinch just inside the separatrix. The region of strong inward pinch agrees very well with the region of strong electron density gradient, suggesting that the inward pinch could be driven by the ion density gradient, as predicted by neoclassical theory. Simulations using the neoclassical impurity convection profile agree very well with experiments. Transport modelling shows that the x-ray pedestal width is largely determined by the diffusion coefficient in the transport barrier. This allows us to diagnose changes in the edge diffusion coefficient on the basis of observations of x-ray pedestal width changes. Significant differences in the edge diffusion coefficient are seen between different types of H-modes. Several scalings for the edge diffusion coefficient in the Enhanced $D_{\alpha} \mathrm{H}$-mode are also identified. This may help us understand the physical processes responsible for this attractive confinement mode.
\end{abstract}




\section{Introduction}

In tokamak fusion experiments, a large reduction in cross-field transport of heat and particles is seen at the transition from the low confinement mode (L-mode) to the high confinement mode (H-mode) [1]. The most dramatic increase in confinement occurs in a narrow region near the last closed flux surface, where the turbulent transport is suppressed. This region is referred to as the transport barrier, or the pedestal region, because the radial profiles of various plasma quantities become pedestal-like as large gradients develop in the transport barrier region. A highly desirable feature of the H-mode is that the global energy confinement time is 2-3 times larger than in L-mode. An undesirable feature of the H-mode is that the impurity ion confinement time can be as much as 50 times larger than in L-mode, resulting in an accumulation of impurities. It is therefore important to understand the physics of the $\mathrm{H}$-mode, and the transport of impurities in the H-mode pedestal region. Several different ways to degrade the impurity confinement, without significantly degrading the energy confinement, have been found. A particularly attractive type of H-mode was discovered in the Alcator C-Mod tokamak, named the Enhanced $D_{\alpha}$ (EDA) H-mode [2]. This mode has much lower impurity confinement than the standard (ELM-free) H-mode, but still retains good energy confinement. It is an important goal of the Alcator C-Mod program to understand the physics of the H-mode transport barrier in the high field, high density shaped tokamak regime unique for this device, in particular the EDA H-mode. Several edge diagnostics have been installed in the last few years on Alcator C-Mod, leading to much improved measurements of the transport barrier region. This paper presents analysis of the radial impurity transport coefficients in the H-mode pedestal region in Alcator C-Mod on a millimeter spatial scale. We base our analysis on highly resolved profiles of soft x-ray emissivity, electron density $\left(n_{e}\right)$, and electron temperature $\left(T_{e}\right)$. In H-mode, the soft x-ray emissivity at the outboard plasma edge develops a distinct pedestal, located well inside the separatrix. The width of this pedestal has been observed to depend on the type of H-mode, and also shows clear scalings with certain plasma parameters [3], [4]. It has been speculated that the x-ray pedestal width reflects the transport properties of the H-mode pedestal region, and that its location well inside the separatrix shows that the impurities are confined further into the plasma because of the inward impurity 
pinch near the plasma edge [5]. The analysis presented in this paper, which has been made possible by the recent addition of a high resolution electron density diagnostic covering the pedestal region, basically confirms these ideas, and provides a detailed understanding of the soft x-ray measurements and the H-mode edge radial transport of impurities. The diagnostics used to obtain $n_{e}, T_{e}$, and soft x-ray emissivity profiles are described in Section 2. H-mode measurements, presented in Section 3, show that the soft x-ray emissivity pedestal is located further into the plasma than the electron density pedestal, which implies that the impurity density pedestal is shifted inward compared to the electron density pedestal. We compare measured x-ray emissivity profiles and profiles calculated on the basis of modelling of the fluorine transport and radiation in Section 4, and find that the inward shift of the x-ray emissivity pedestal is due to an inward shift of the impurity (fluorine) pedestal. Measurements of the width and the location of the soft x-ray pedestal yield information about the radial profiles of the diffusion coefficient $D$ and the impurity convection velocity $v$. We find evidence for a strong inward convection of impurities localized to the electron density pedestal region, in agreement with neoclassical theory. Modelling also shows that the x-ray pedestal width can be used to estimate $D$ in the transport barrier region, allowing us to diagnose changes in the transport barrier between various types of $\mathrm{H}$-modes as well as identifying scaling laws for $D$ in the EDA H-mode. These results are presented in Section 5. Finally, we summarize our results in Section 6.

\section{Edge diagnostics}

The impurity transport analysis presented in this paper is based on measurements of soft x-ray emissivity, electron density, and electron temperature. The soft x-ray emissivity is measured with $1.5 \mathrm{~mm}$ radial resolution at the outboard edge by an array of 38 photodiode detectors viewing the plasma edge through a $10 \mu \mathrm{m}$ beryllium foil. During the 1997-1998 run campaign, a soft x-ray array viewed the lower part of the outboard edge from above. Details of that diagnostic and the analysis methods used can be found in Ref. [3]. During the 1999 run campaign, a second array viewed the upper part of the outboard edge from the bottom in a configuration which is essentially the mirror image of the previous configuration. All results 
from the new configuration agree with previous findings from the old configuration, and the same analysis methods were employed.

The electron temperature $T_{e}$ is derived from electron cyclotron emission (ECE) measurements at the plasma midplane. High resolution edge profiles of $T_{e}$ are obtained by sweeping the toroidal field by a small amount, moving the ECE resonance of a given frequency across the pedestal. The spatial resolution is about $9 \mathrm{~mm}$ when this technique is employed [6]. The electron density is derived from a newly installed visible continuum diagnostic which measures plasma emission in a spectral band around $536 \mathrm{~nm}$ with FWHM of $3 \mathrm{~nm}$. By using the temperature profiles from the ECE measurements, one can account for the weak temperature dependence of the radiation and derive the quantity $n_{e} \sqrt{Z_{e f f}}[7]$, [8]. In Alcator C-Mod, $Z_{\text {eff }} \approx 1.2$ under normal operating conditions, so the variation in $\sqrt{Z_{\text {eff }}}$ is very small. Thus, the $n_{e}$ profile can be derived with good accuracy. The measurements are made along tangential chords which are $1 \mathrm{~cm}$ below the plasma midplane with about $2.5 \mathrm{~mm}$ radial resolution in the edge region. A schematic of the views of the three diagnostics is shown in Figure 1. The measurements of the three diagnostics are compared by mapping the soft x-ray measurements back to the plasma midplane using the EFIT flux surfaces. Because all three measurements are made very close to or at the plasma midplane, this process is very insensitive to any magnetic field line mapping uncertainties, including any uncertainty in the exact location of the separatrix. A second soft x-ray array views the top of the plasma; results from this diagnostic will be the subject of a future publication.

\section{$3 \quad$ Measurements of radial profiles}

In Figure 2 we show measured radial profiles of electron density, temperature, and soft x-ray emissivity for an ELM-free ohmic H-mode. The soft x-ray emissivity shows a distinct and narrow pedestal in ELM-free H-modes, less than $2 \mathrm{~mm}$ wide, as defined by the full width of the tanh-like function commonly used to characterize the H-mode pedestal shapes [9]:

$$
A \times \frac{1}{2}\left(\tanh \left(\frac{r_{p e d}-r}{\Delta / 2}\right)+1\right)+B \times\left(r_{p e d}-\Delta / 2-r\right) \times \Theta\left(r_{p e d}-\Delta / 2-r\right)
$$

Here, $A$ is the amplitude of the pedestal, $B$ is the linear slope inside the pedestal, $r_{p e d}$ is the location of the center of the pedestal, $\Delta$ is the full pedestal width, and $\Theta$ is the Heaviside 
step function. The foot of the soft x-ray pedestal, which can be defined in terms of the tanh-pedestal parameters as $r_{p e d}+\Delta / 2$, is located about $10 \mathrm{~mm}$ inside the separatrix. These measurements are typical of ELM-free H-modes, irrespective of whether they were obtained with or without auxiliary radio frequency heating. In contrast to the soft x-ray pedestal, the feet of the pedestals in electron temperature and density are located about $2 \mathrm{~mm}$ inside the EFIT separatrix in this case, and both of these pedestals are significantly wider (about $8 \mathrm{~mm}$ ) than the soft x-ray emissivity pedestal. The electron density pedestal can be much narrower in some H-modes, but the electron temperature pedestal width measured by ECE is usually in the range of $8-15 \mathrm{~mm}$, barely resolvable with the $8 \mathrm{~mm}$ spatial resolution. The soft x-ray pedestal width typically varies in the range from 1-6 mm, depending on the type of H-mode and on several plasma parameters, as discussed in more detail in Section 5. The narrowest pedestals, whose widths are barely resolvable at the $1.5 \mathrm{~mm}$ radial resolution, are seen during ELM-free H-modes, and the widest are seen during type III ELMy periods and low current EDA H-modes. Regardless of the type of H-mode, the soft x-ray pedestal is always located well inside the pedestals of electron density and temperature. In Figure 3 we show the location of the foot of the visible continuum emissivity pedestal plotted against the location of the foot of the soft x-ray pedestal at the outboard edge, for 112 different discharges taken from a database of edge pedestal parameters. The two locations are very well correlated, but the foot of the soft x-ray pedestal is on average $9 \mathrm{~mm}$ inside the foot of the visible continuum emissivity pedestal. The visible continuum emissivity pedestal width and location, available routinely on many discharges, are good approximations to the electron density pedestal width and location, which are only available for a relatively small number of discharges. Previous rudimentary calculations indicated that a major contribution to the soft x-ray emissivity is proportional to $n_{F} \times n_{e}$ where $n_{F}$ is the density of fully ionized fluorine, and that the temperature dependence is small [3]. More detailed calculations, presented in Section 4 take the temperature effects explicitly into account. Due to strong line radiation, the temperature effects are not as small as previously thought. Fluorine is an intrinsic impurity in Alcator C-Mod, presumably originating from teflon insulation or solvents. Recently, injections of $\mathrm{CaF}_{2}$ and freon gas have confirmed that the x-ray emissivity increases with the fluorine density. With the recent addition of the visible continuum emissivity diagnostic, highly resolved profiles of electron density are now 
available, and they allow us to confirm that the steep pedestal in soft x-ray emissivity is due almost entirely to a steep pedestal in fluorine density, and that the density of fluorine must be very low in the region between the foot of the x-ray pedestal and the foot of the electron density pedestal, since there is appreciable electron density, but hardly any soft x-ray emission in this region.

\section{Modelling of impurity radiation and transport}

\subsection{Comparisons between modelling and experiments}

It follows from the experimental observations presented in Section 3 that the impurities at the outboard edge are confined further inside the transport barrier than the bulk plasma. This strongly suggests the existence of an inward convection of impurities in the region 0-10 $\mathrm{mm}$ inside the separatrix. A strong inward convection of impurities localized to the edge region has previously been measured in Alcator C-Mod H-modes [10] as well as on several other tokamaks, such as ASDEX [11], DIII-D [12], and JET [13]. Such an inward impurity pinch could be driven by the large ion density gradient near the edge, as predicted by neoclassical theory. The neoclassical prediction of the inward impurity pinch is, in the limit of trace impurities relevant to Alcator C-Mod [14]:

$$
v_{\text {neoc }}=\nu_{i} q^{2} \rho_{i}^{2}\left(Z_{I} \frac{1}{n_{i}} \frac{\partial n_{i}}{\partial r}-\frac{1}{T_{i}} \frac{\partial T_{i}}{\partial r}\right)
$$

where $\nu_{i}$ is the ion-ion collision frequency, $q$ is the safety factor, $\rho_{i}$ is the ion Larmor radius, $Z_{I}$ is the charge of the impurity ion, $n_{i}$ is the ion density, and $T_{i}$ is the ion temperature, assumed equal to the impurity temperature. This formula is derived in a cylindrical geometry and assumes that the poloidal ion gyroradius is much smaller than the gradient scale lengths. This latter condition is usually not fulfilled in Alcator C-Mod H-modes. It also assumes that the poloidal variations in plasma parameters are small. In fact, comparisons between the x-ray emissivity at the top and the outboard edge of the plasma show that there are large poloidal variations in impurity density near the separatrix. In this paper, we ignore these complications, which will be addressed in a future publication. We consider impurity transport to be a purely one-dimensional (radial) process. For medium and high $\mathrm{Z}$ impurities, the density gradient 
term dominates in the transport barrier region, leading to an inward convection. On Alcator C-Mod, high resolution ion density and temperature measurements are not available in the pedestal region. However, because $Z_{\text {eff }} \approx 1$, quasi-neutrality leads to $n_{i} \approx n_{e}$. We also assume $T_{i} \approx T_{e}$, which is believed to be a good approximation because of the high electron-ion collision frequency in the high density H-mode edge in Alcator C-Mod. Thus, we can use the electron measurements to estimate the neoclassical convection term. For the plasma profiles presented in Figure 2 the neoclassical convection profile for fully ionized fluorine is shown in Figure 4. There is a large inward pinch confined to a narrow region close to the separatrix. The MIST code [15] is used to calculate the steady state density profiles for all the charge states of fluorine. As inputs to the code we use the measured $n_{e}$ and $T_{e}$ profiles, the neoclassical convection velocity, and a simple profile for the diffusion coefficient $D$, shown in Figure 5 . This profile, which has a value of $0.01 \mathrm{~m}^{2} / \mathrm{s}$ in the edge transport barrier region, and $0.2 \mathrm{~m}^{2} / \mathrm{s}$ in the core region, is similar to previous experimentally derived $D$ profiles for ELM-free H-modes [10]. The steady state profiles of the various charge states of fluorine calculated by the MIST code are then used together with the electron density and temperature profiles to calculate profiles of emission from fluorine line radiation and recombination radiation, as well as bulk plasma bremsstrahlung. The spectral distributions of these processes are convolved with the transmission function for the $10 \mu \mathrm{m}$ beryllium foil and added up, yielding a profile of soft $\mathrm{x}$ ray emissivity which can be directly compared to the measured profile. This method directly incorporates the effects of $T_{e}$ and $n_{e}$. Although the temperature dependence is somewhat stronger than was concluded on the basis of less detailed calculations [3], it remains relatively weak over the region where the soft x-ray pedestal is located. In Figure 6 we show the radial profiles of the three highest charge states of fluorine, as calculated by MIST, and below we show the calculated soft x-ray emissivity, compared to the measured profile, showing excellent agreement. It is confirmed that the soft x-ray emissivity profile closely resembles the profiles of the densities of the high charge states of fluorine. The central fluorine density is treated as a free variable, chosen in this case to be $0.14 \%$ of the central electron density in order to match the absolute value of the measured x-ray emissivity profile. Thus, we can derive absolutely calibrated profiles of the fluorine density. As illustrated in Figure 6, most of the soft x-ray emission comes from line radiation, with a smaller but significant contribution from 
recombination of fully ionized fluorine. The dominant line is that of Lyman $\alpha$ (the $\mathrm{n}=2$ to 1 transition of hydrogen-like fluorine at $\lambda=14.98 \AA$ ). Originally, line radiation was thought to be less important but these new detailed simulations show that line radiation accounts for more than half of the soft x-ray emission from fluorine, and therefore the profile of hydrogenlike fluorine is at least as important to the soft x-ray emissivity profile as the profile of fully ionized fluorine. The bremsstrahlung radiation from the main plasma is unimportant because most of the bremsstrahlung photons are not energetic enough to penetrate the beryllium filter at the rather low temperatures characteristic of the edge region. We conclude that modelling of fluorine transport and radiation, using a neoclassical inward pinch combined with a simple empirical profile for $D$, and the measured profiles for $T_{e}$ and $n_{e}$, yields profiles of soft x-ray emissivity which are remarkably similar to the measured profiles. An inward pinch profile consistent with neoclassical theory has been reported in H-mode discharges on several other tokamaks, including DIII-D [12], and ASDEX [11]. Modelling of the fluorine transport with no inward pinch results in profiles of soft x-ray emissivity which are much wider (about 10 $\mathrm{mm}$ ) and closer to the separatrix than the measured soft x-ray emissivity profiles.

\subsection{Modelling of other impurities}

A variety of different impurities has been injected into H-mode discharges in Alcator C-Mod, and with the exceptions of neon and fluorine, none of these leads to appreciable increases in the measured edge soft x-ray emissivity [3]. Oxygen, which is an intrinsic impurity, may contribute somewhat to the x-ray emissivity, but since Alcator C-Mod is regularly boronized, the oxygen levels are generally too low to contribute significantly to the soft x-ray emissivity. We have performed modelling of the oxygen and neon transport and radiation processes, and have found that the soft x-ray emissivity profiles originating from oxygen and neon are very similar in shape to those from fluorine, when the same profiles of $n_{e}, T_{e}, D$, and $v=v_{\text {neoc }}$ are used in the modelling. We find that when the beryllium filter function has been taken into account, x-ray emissivity levels from a given density of oxygen would be about one third of the x-ray emissivity levels from the same density of fluorine. For oxygen, the dominant contribution of $\mathrm{x}$-ray emission comes from recombination of the fully stripped ion.

Modelling of neon transport and radiation processes shows that the soft x-ray emissivity trans- 
mitted through the beryllium filter leads to levels three times larger than the same amount of fluorine. For neon, the dominant source of x-ray emissivity through the beryllium filter is line radiation, mostly from the Lyman $\alpha$ line of hydrogen-like neon at $\lambda=12.13 \AA$. The neon Lyman $\alpha$ line is much stronger than the fluorine Lyman $\alpha$ because a larger fraction of the neon is in the hydrogen-like state, and because the Lyman $\alpha$ line for neon is at a higher photon energy than the fluorine Lyman $\alpha$ line, so that a larger fraction of it is transmitted through the beryllium filter. These results agree well with the experimental observation that even rather modest injections of neon can increase the soft x-ray emissivity by factors of 2-5. After such an injection, the x-ray emissivity profile shape remains the same as before the neon injection with respect to the width and location of the soft x-ray emissivity pedestal, also in agreement with the modelling. Neon is not an intrinsic impurity, so it contributes to the x-ray emissivity only during discharges where it is actively injected. No oxygen injections were performed during the operation of the edge soft x-ray arrays, so their sensitivity to the oxygen content has not been directly confirmed in experiments.

\subsection{Sensitivity study}

A sensitivity study has been performed to investigate the effects of $D$ and $v$ on the x-ray pedestal, calculated on the basis of the MIST simulations. In this study, a narrow, triangular shape for $v$ was used, peaking at $-100 \mathrm{~m} / \mathrm{s}$, similar in shape to the neoclassical inward pinch, as seen in Figure 7. The location of this pinch, as defined on the figure, was then varied. For $D$, the core value was kept constant, but several different values for the edge region were used, as shown in Figure 8. The edge values were chosen to span the range measured previously in injection experiments for the three types of H-modes seen on Alcator C-Mod (ELM-free, Enhanced $D_{\alpha}$, and type III ELMy H-modes). These simulations show that when the inward pinch is located 10-20 mm inside the EFIT separatrix, regardless of the value of $D$, the location of the inward pinch coincides with the following location

$$
R_{x t o p}=R_{x p e d}-0.4 \Delta_{X}
$$

Here, $R_{x p e d}$ is the center position, and $\Delta_{X}$ is the full width of the computed soft x-ray pedestal, as defined by the tanh-like fit in Equation 1. $R_{x t o p}$ is located just below the top of the x-ray 
pedestal, regardless of the width. $R_{x t o p}$, as defined above, is typically $11-16 \mathrm{~mm}$ inside the EFIT separatrix in actual experiments, so we infer that the innermost point of inward pinch is also typically 11-16 mm inside the EFIT separatrix. From neoclassical theory, we expect that the innermost point of the region of strong inward pinch should be located at the innermost point of large electron density gradient, that is, at the top of the electron density pedestal. We have investigated if this is generally the case for measured profiles, using the database of pedestal parameters which includes both the soft x-ray pedestal measurements and the visible continuum emissivity pedestal measurements for a large number of discharges. As mentioned, the visible continuum emissivity pedestal provides a reasonable approximation to the electron density pedestal. We define the top of the visible continuum emissivity pedestal, $R_{v c t o p}$, as:

$$
R_{v c t o p}=R_{v c p e d}-\Delta_{v c}
$$

Here, $R_{v c p e d}$ and $\Delta_{v c}$ are the fitted central position and width of the visible continuum emissivity pedestal. In Figure 9 we plot $R_{x t o p}$ versus $R_{v c t o p}$ for a large number of plasma discharges from the database. The Figure shows a clear correlation between the two locations, with a systematic shift of about $2 \mathrm{~mm}$. This supports our conclusion that the x-ray pedestal location is determined by the location of the inward pinch, which in turn is determined by the location of the plasma density pedestal.

The width of the calculated x-ray pedestal is determined by the edge value of $D$. Edge values of $D$ around $0.01 \mathrm{~m}^{2} / \mathrm{s}$, corresponding to previous measurements in ELM-free H-mode, yield xray pedestal widths of $1.5-2 \mathrm{~mm}$, whereas edge values of $D$ around $0.05 \mathrm{~m}^{2} / \mathrm{s}$, corresponding to type III ELMy H-mode values, yield x-ray pedestal widths around $4.2 \mathrm{~mm}$. These predictions are in good agreement with measurements of the soft x-ray pedestal width during ELM-free and type III ELMy H-modes, as discussed in Section 5. In Figure 10 we plot the simulated soft x-ray emissivity pedestal width as a function of the transport barrier diffusion coefficient, as derived from the sensitivity study just described. We also performed a similar parameter scan with a triangular pinch of the same width, but peaking at $-300 \mathrm{~m} / \mathrm{s}$ instead of $-100 \mathrm{~m} / \mathrm{s}$. The results were similar, except that the calculated x-ray pedestal widths were somewhat smaller than those quoted here, because of the steeper slope of the pinch profile. They still showed a clear tendency for the x-ray pedestal width to increase with increasing edge $D$. The peak value of the inward pinch cannot be determined from the steady-state profiles of x-ray emissivity, 
since the inward pinch peaks in a region where there is essentially no highly ionized fluorine, and thus very low x-ray emissivity. Impurity injection experiments should be able to address this question.

\section{Interpretation of x-ray pedestal width measure- ments}

\subsection{Differences in the diffusion coefficient in different types of H-modes}

In previous publications, it was noted that the soft x-ray emissivity pedestal width at the outboard edge is significantly larger in the EDA H-mode $(3-7 \mathrm{~mm}$, depending on the plasma current) than in ELM-free H-mode (1.5-3 mm) [3]. We have also consistently seen that during type III ELMy H-modes with relatively poor confinement, the x-ray pedestal becomes wide (5 $\mathrm{mm}$ or more). The sensitivity analysis allows us to interpret these measurements and conclude that the diffusion coefficient in the transport barrier is significantly larger in the EDA Hmode than in ELM-free H-mode, and even larger in type III ELMy H-mode. This agrees well with previous independent measurements of the edge diffusion coefficient, as well as with the general observation that the distinguishing characteristic of the EDA H-mode is that it has a much lower particle confinement time than the ELM-free H-mode [2]. Type III ELMy Hmodes, which on Alcator C-Mod occur only in H-modes near the H-L threshold, are frequently observed to have only marginally better energy and particle confinement than L-modes.

\subsection{Scalings of the diffusion coefficient in EDA H-mode}

Several empirical scaling laws for the soft x-ray pedestal width have been found in the EDA H-mode. The soft x-ray pedestal width is an increasing function of the inverse of the plasma current, $1 / I_{p}$. The observed scaling with $1 / I_{p}$, which is stronger than linear, could also be a scaling with the safety factor at the $95 \%$ flux surface, $q_{95}$, since the two are strongly correlated

when the toroidal magnetic field is kept constant. The toroidal field is usually kept near 5.2 
Tesla in Alcator C-Mod to allow for efficient on-axis RF heating, and most of the EDA H-modes have been obtained at toroidal fields very close to this value. A few ohmically heated EDA H-modes were obtained at lower toroidal fields but the measured x-ray pedestal widths did not conclusively show if $I_{p}$ or $q_{95}$ is the relevant scaling parameter. A limited sweep of toroidal field from 5.0 to $6.0 \mathrm{~T}$ during a single discharge with constant $I_{p}$ showed no measurable change in the x-ray pedestal width. This suggests that $1 / I_{p}$ is more important than $q_{95}$, but it is not conclusive evidence because the toroidal field was only changed by $20 \%$. Future upgrades of the RF system will enable efficient heating at a variety of toroidal fields, which may allow us to address this question better. An approximately linear scaling of the soft x-ray pedestal width with the triangularity has also been observed. On the basis of these results, we conclude that the edge diffusivity is an increasing function of the triangularity and of $1 / I_{p}$ (or $q_{95}$ ). This suggests that the quasi-coherent fluctuations observed in EDA H-mode, which are believed to be causing the increased diffusion in the transport barrier, are more unstable at low plasma current (high $q_{95}$ ) and high triangularity. This is consistent with previous results showing that high $q_{95}$ and moderate to high triangularity tend to favor the EDA H-mode over the ELM-free $\mathrm{H}$-mode [2]. We also note that previous injection experiments in L-mode in Alcator $\mathrm{C}$ showed that $D$ is an increasing function of $q$ at the plasma edge, rather than $1 / I_{p}[16]$. The pedestal database has also been used to identify a third parameter affecting the x-ray pedestal width, and therefore the diffusion coefficient in the transport barrier region, namely $d I_{p} / d t$. Ramping the current up tends to increase the x-ray pedestal width, whereas ramping the current down tends to decrease it, as compared to pedestal widths at the same constant plasma current. We illustrate this in Figure 11. This result has yet to be confirmed in a dedicated series of experiments. It is not caused by a time lag in the response of the soft x-ray pedestal width to the change in $I_{p}$. It implies that the diffusion coefficient in the transport barrier is larger when the current is being ramped up than when the current is ramped down. One should therefore expect that the impurity confinement time is lower when the current is ramping up. It also suggests that the fluctuations in EDA are sensitive to relatively small changes in the edge current density profile, which presumably may be affected by the direction of the ramp. We also speculate that it may be easier to obtain EDA H-modes when ramping the current up than when the current is kept steady or is being ramped down, since we have generally 
observed that parameters favoring wide x-ray pedestals also favor the EDA H-mode over the ELM-free H-mode. Experiments to test this are planned.

\section{Summary}

By combining highly resolved measurements of the soft x-ray emissivity, electron density, and electron temperature in the region just inside the separatrix, it is possible to do detailed measurements of impurity transport coefficients in the H-mode transport barrier. Our results show the existence of an inward pinch, whose location coincides with the region of large plasma density gradients, as predicted from neoclassical theory. Simulations using the neoclassical pinch profile yield results which are in excellent agreement with the measured soft x-ray emissivity profiles. The diffusion coefficient in the transport barrier region, which is much reduced compared to the core region, determines the soft x-ray pedestal width, although the inward pinch profile also affects the width to some degree. Measurements of the soft x-ray pedestal width can therefore be used to estimate the transport barrier diffusion coefficient. In this way, we confirm previous findings that the transport barrier diffusion coefficient is very low in ELM-free H-mode, on the order of $0.01 \mathrm{~m}^{2} / \mathrm{s}$ (lower than the neoclassical diffusion coefficient), somewhat larger in EDA H-modes, and largest, near $0.10 \mathrm{~m}^{2} / \mathrm{s}$, in type III ELMy H-modes. We also find that in EDA H-mode, the transport barrier diffusion coefficient increases with triangularity and $q_{95}$ (or $1 / I_{p}$ ). $D$ is also affected by the direction of the current ramp. This latter finding suggests that the quasi-coherent fluctuations, which presumably are driving the enhanced diffusivity in EDA H-mode, are sensitive to the edge profile of the current density, or the magnetic shear.

\section{Acknowledgements}

The authors would like to thank Dr. S. Wolfe for magnetic surface reconstructions, Dr. B. LaBombard and Mr. S. Gangadahra for freon injections, Dr. D. Mossessian and Mr. J. Hughes for Thomson scattering measurements, Dr. S. Wukitch for ICRF heating, Dr. J. Goetz for impurity injections and impurity transport modelling, and Dr. M. Greenwald for 
fruitful discussions about the EDA H-mode. The work at the Alcator C-Mod facility at the Massachusetts Institute of Technology was supported by the U.S. Dept. of Energy, Coop. Agreement DE-FC02-99ER54512.

\section{References}

[1] F. Wagner et al., Phys. Rev. Letters 49, p. 1408 (1982)

[2] M. Greenwald et al., Phys. Plasmas, 6, p. 1943, (1999)

[3] T. Sunn Pedersen and R.S. Granetz, Rev. Sci. Instrum. 70 (1999), p. 586

[4] R.S. Granetz et al., Proceedings from the 17th IAEA Fusion Energy Conference, Yokohama, Japan (1998)

[5] I.H. Hutchinson et al., Plasma Phys. Contr. Fusion 41 (1999) p. A609

[6] A.E. Hubbard et al., Phys. Plasmas 5 (1998), p. 1744

[7] M.E. Foord, E.S. Marmar, J.L. Terry, Rev. Sci. Instrum. 53, (1982), p. 1407

[8] M.E. Foord, E.S. Marmar, Nucl. Fusion 25 (1985), p. 197

[9] R.J. Groebner and T.N. Carlstrom, Plasma Phys. Controlled Fusion 40 (1998) p. 673

[10] J.E. Rice et al., Phys. Plasmas 4 (1997), p. 1605

[11] ASDEX Team, Nucl. Fusion 29 (1989), p. 1959

[12] M.E. Perry, N.H. Brooks, D.A. Content, R.A. Hulse, M. Ali Mahdavi, H.W. Moos, Nucl. Fusion 31 (1991), p. 1859

[13] D. Pasini et al., Plasma Phys. Contr. Fusion 34 (1992), p. 677

[14] P.H. Rutherford, Phys. Fluids 17 (1974), p. 1782

[15] R.A. Hulse, Nuclear Technology/Fusion 3 (1983) p. 259

[16] E.S. Marmar, J.E. Rice, J.L. Terry, F.H. Seguin, Nucl. Fusion 22 (1982), p. 1567 


\section{Figure captions:}

Figure 1: Schematic of the locations of the three diagnostics used in the impurity transport analysis in the Alcator C-Mod vessel. A typical plasma equilibrium is shown for comparison. The ECE diagnostic views a range of radial positions along a major radius. The visible continuum array views tangentially along chords which are $1 \mathrm{~cm}$ below the midplane.

Figure 2: High resolution profiles of $n_{e}, T_{e}$, and x-ray emissivity are shown for an ELM-free H-mode. The $n_{e}$ and $T_{e}$ profiles are idealized fitted profiles closely resembling the measured profiles.

Figure 3: The foot of the soft x-ray pedestal is located on average $9 \mathrm{~mm}$ inside the foot of the visible continuum emissivity pedestal, for a wide range of pedestal locations. Figure 4: The neoclassical impurity convection profile as calculated from the smoothed profiles of $n_{e}$ and $T_{e}$ in Figure 2. The separatrix is located at $\mathrm{r}=0.214 \mathrm{~m}$.

Figure 5: The profile for the diffusion coefficient $D$ used in the MIST modelling.

Figure 6: The upper graph shows the density profiles for the three highest charge states of fluorine, as calculated by the MIST code. The lower graph shows the x-ray emissivity calculated on the basis of the MIST results, compared to the measured x-ray emissivity. The lower graph also shows the separate contributions from line radiation and recombination of fully ionized fluorine.

Figure 7: The triangular pinch profile used in the sensitivity study. The location of the pinch is defined as the innermost point of the triangular shape, as shown on the figure. The profile shown here is located $14 \mathrm{~mm}$ inside the separatrix.

Figure 8: Several different profiles for $D$ were used in the sensitivity study, spanning a range of different values in transport barrier region, but with the same core value.

Figure 9: This figure shows the correlation between $R_{x t o p}$ and $R_{v c t o p}$ for 1463 pedestal measurements from 112 different discharges. Binned averages of $R_{v c t o p}$ are also shown, each with a vertical bar indicating the standard deviation in each bin.

Figure 10: This figure shows the correlation between the diffusion coefficient and the x-ray pedestal width, derived from the sensitivity study using the $D$ and $v$ profiles from Figures 7 and 8 . 
Figure 11: This figure shows the soft x-ray pedestal width as a function of plasma current, for EDA H-modes in which the current was either being ramped up or ramped down. 174 pedestal measurements from 38 different discharges are included, showing the familiar scaling with plasma current, but also that the ramp-up discharges have larger pedestal widths than the ramp-down discharges. 


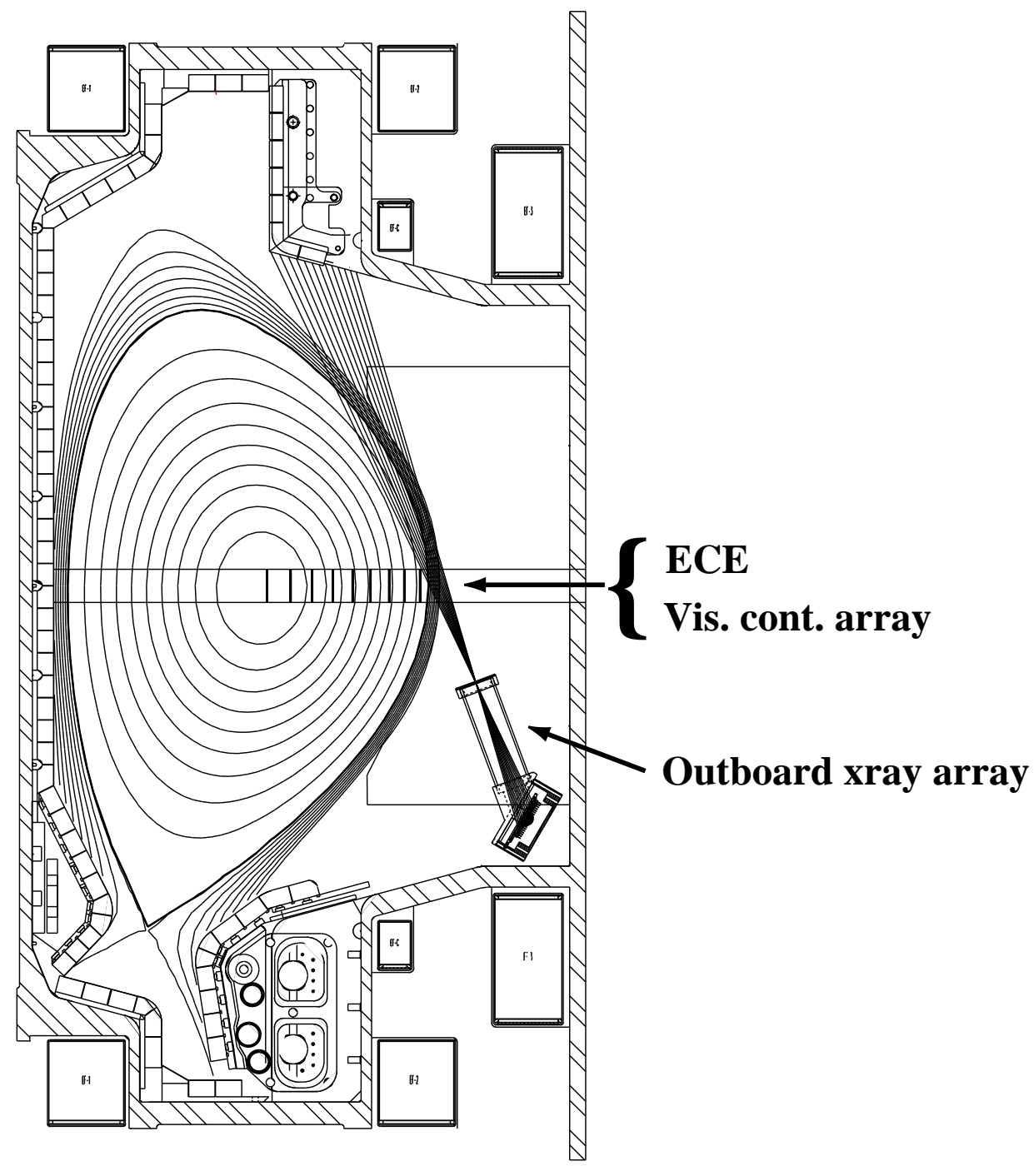

Figure 1: 


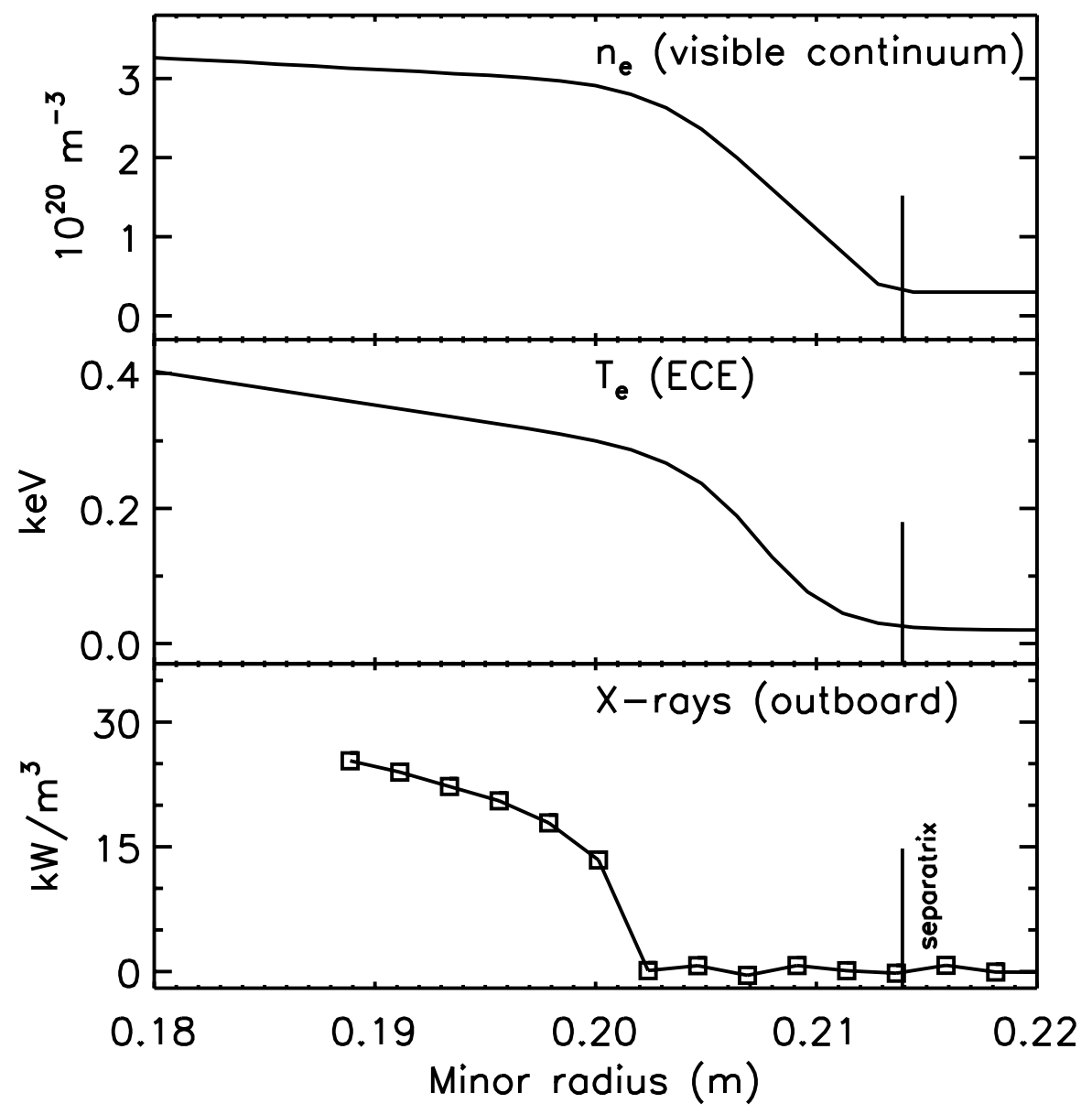

Figure 2: 


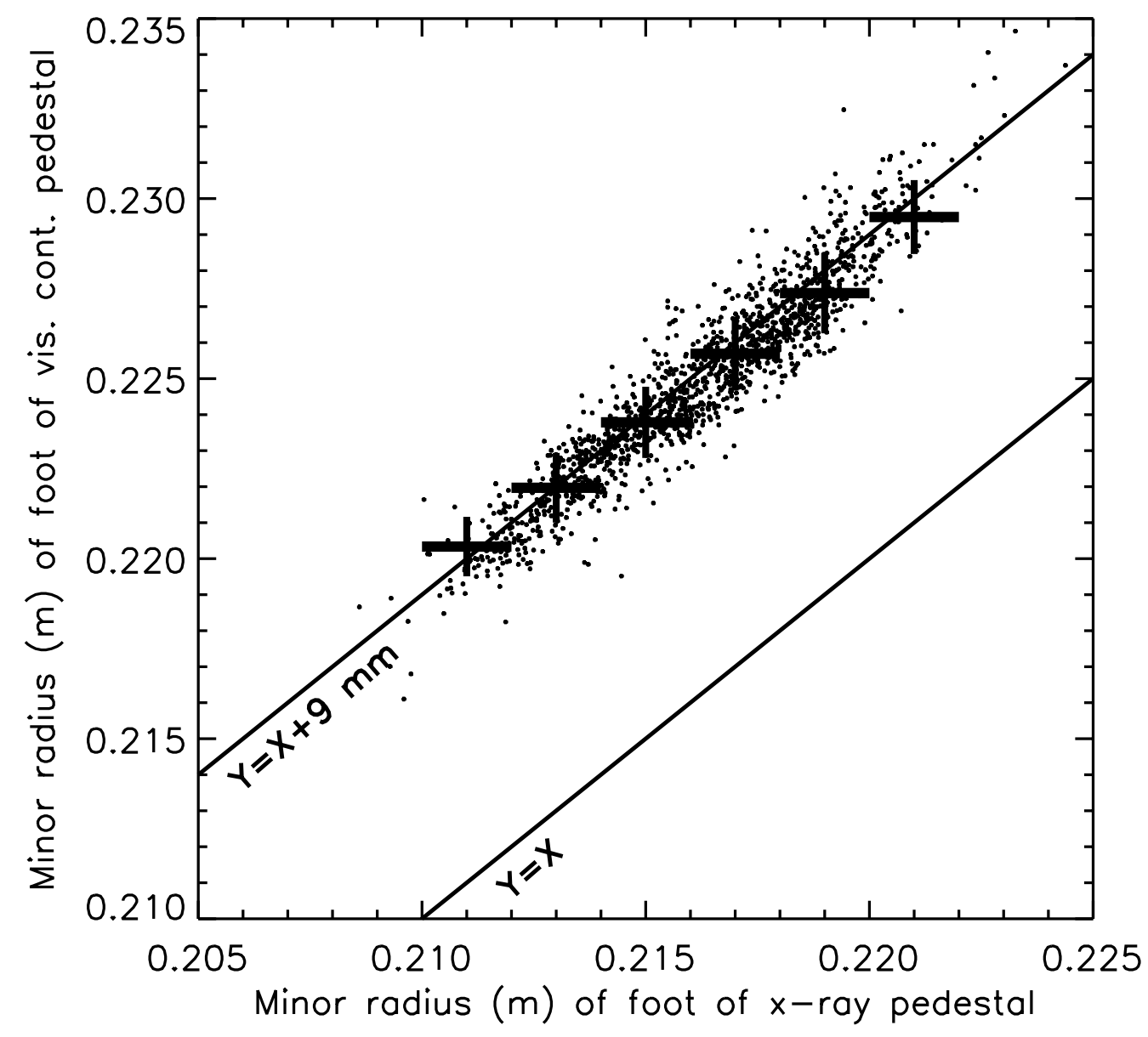

Figure 3: 


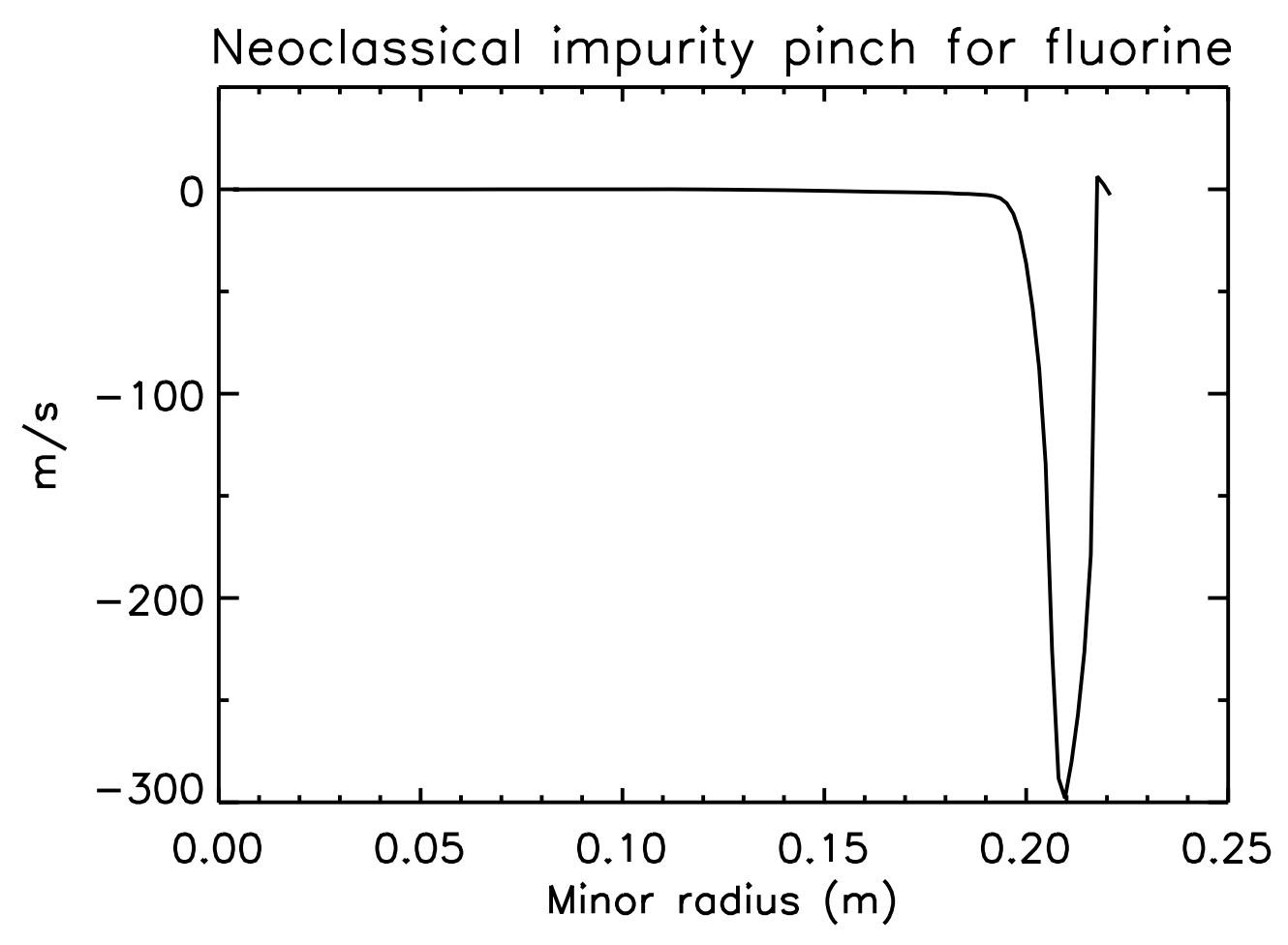

Figure 4: 


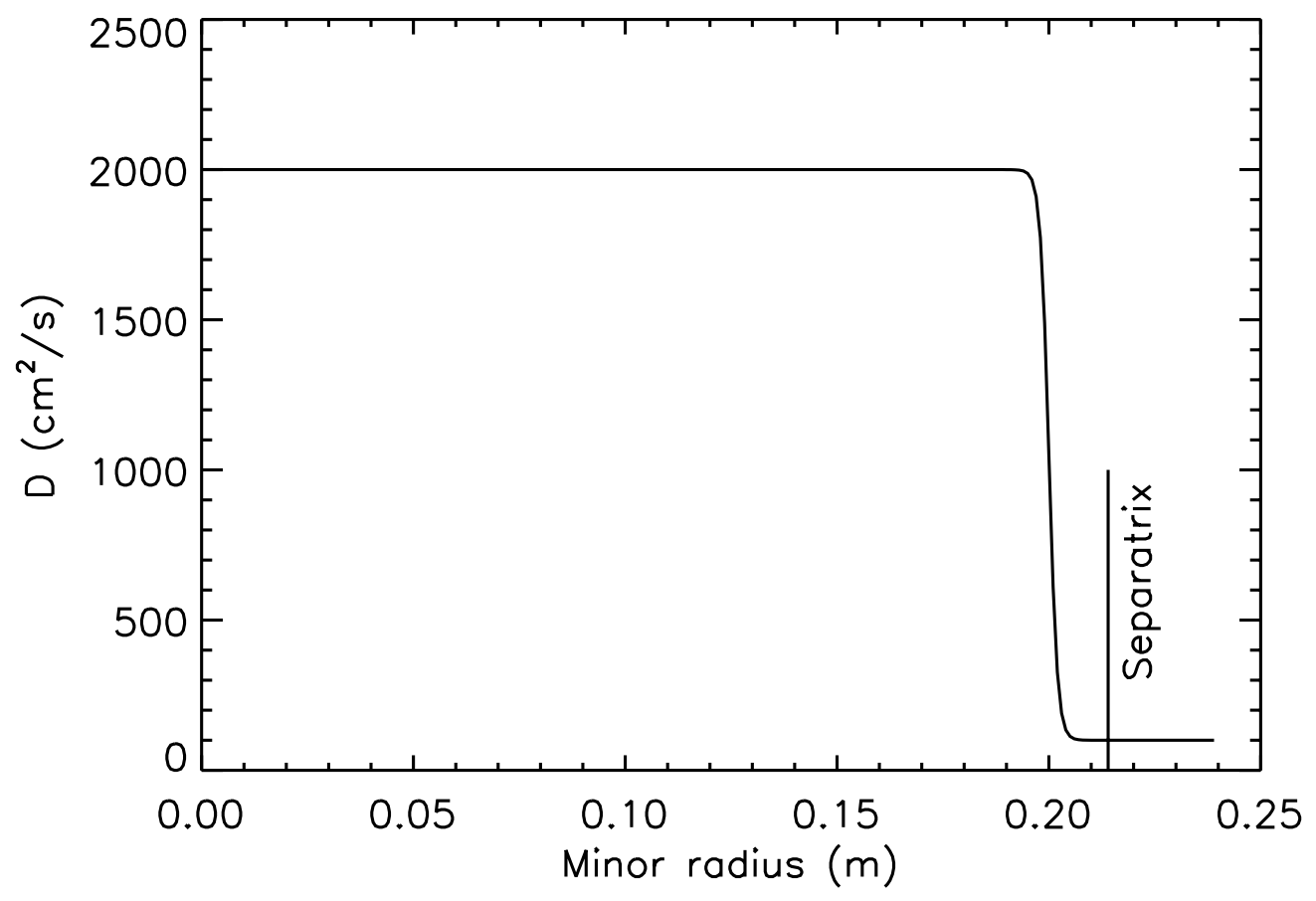

Figure 5: 


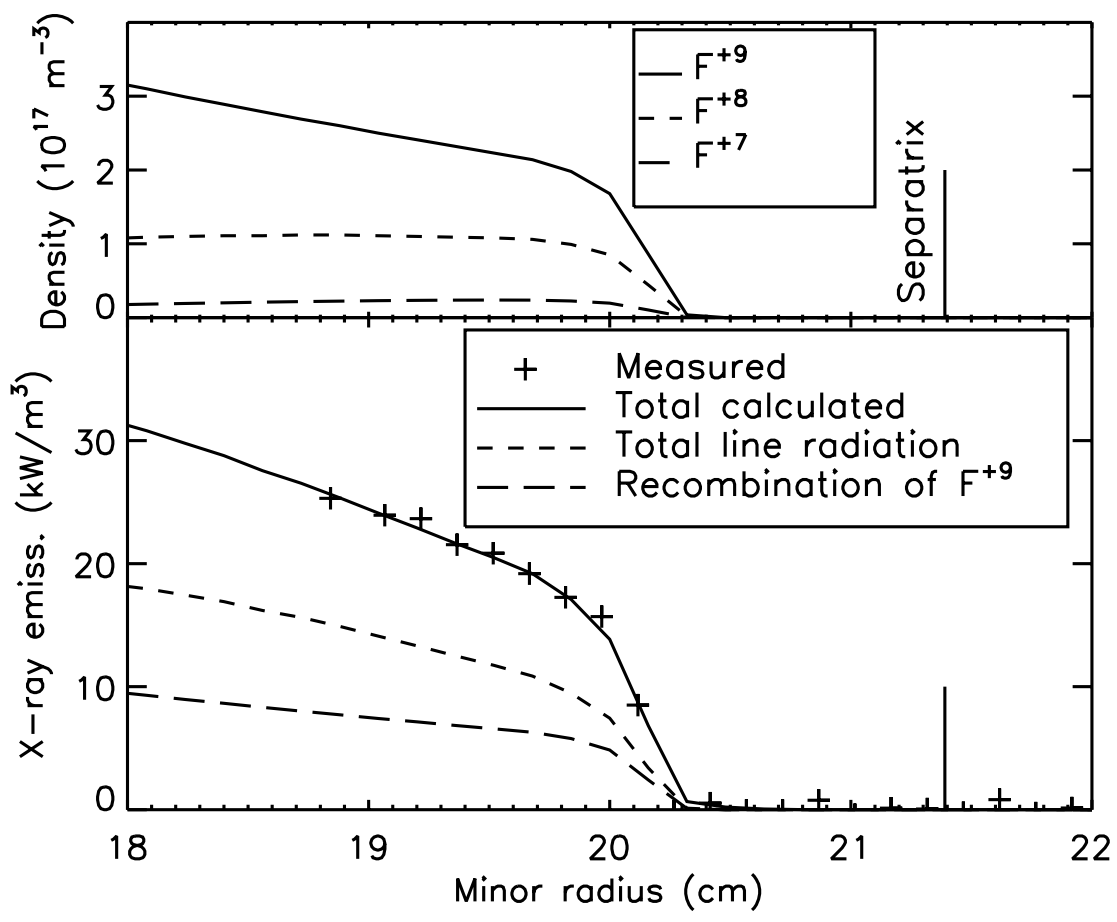

Figure 6: 


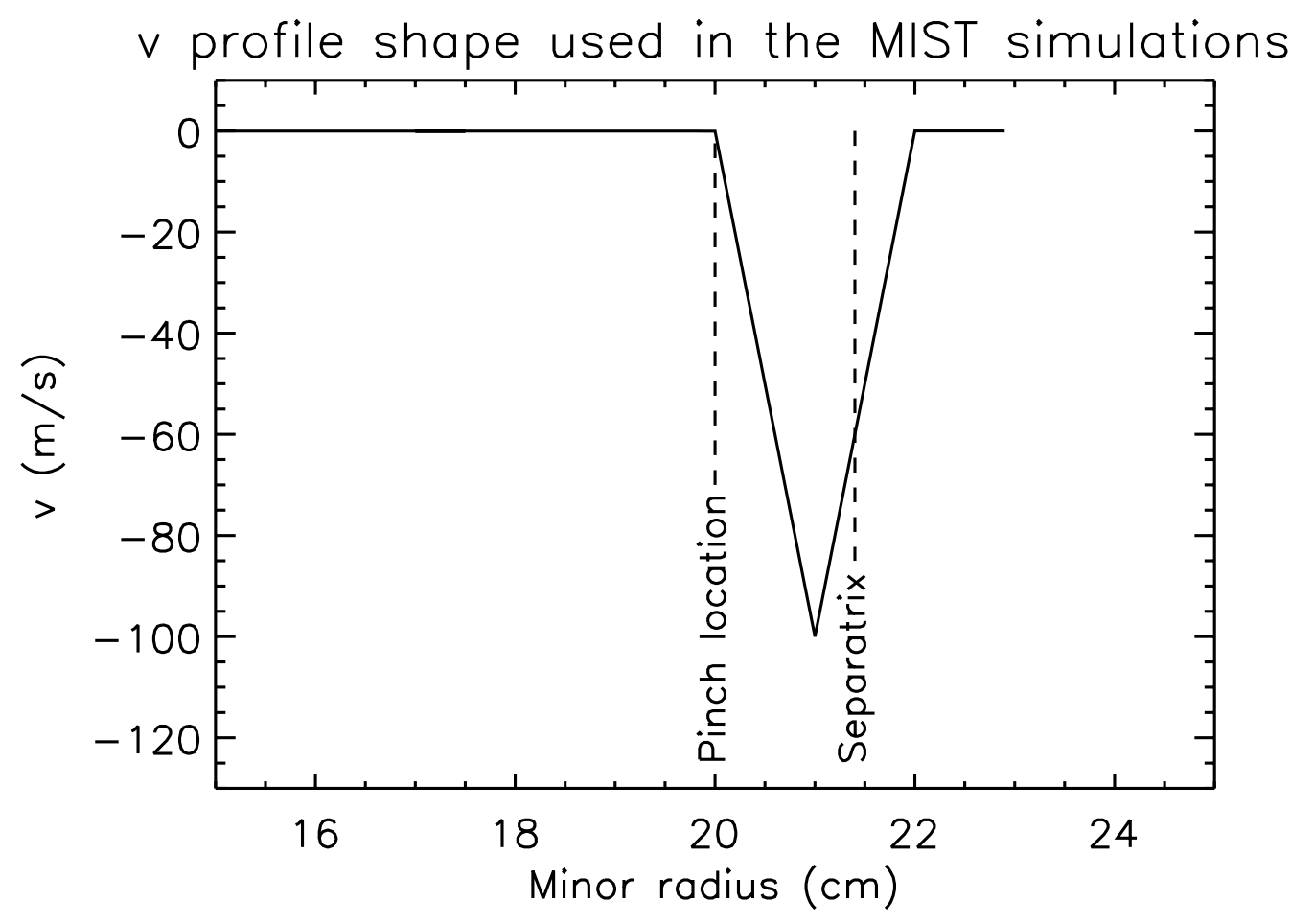

Figure 7: 


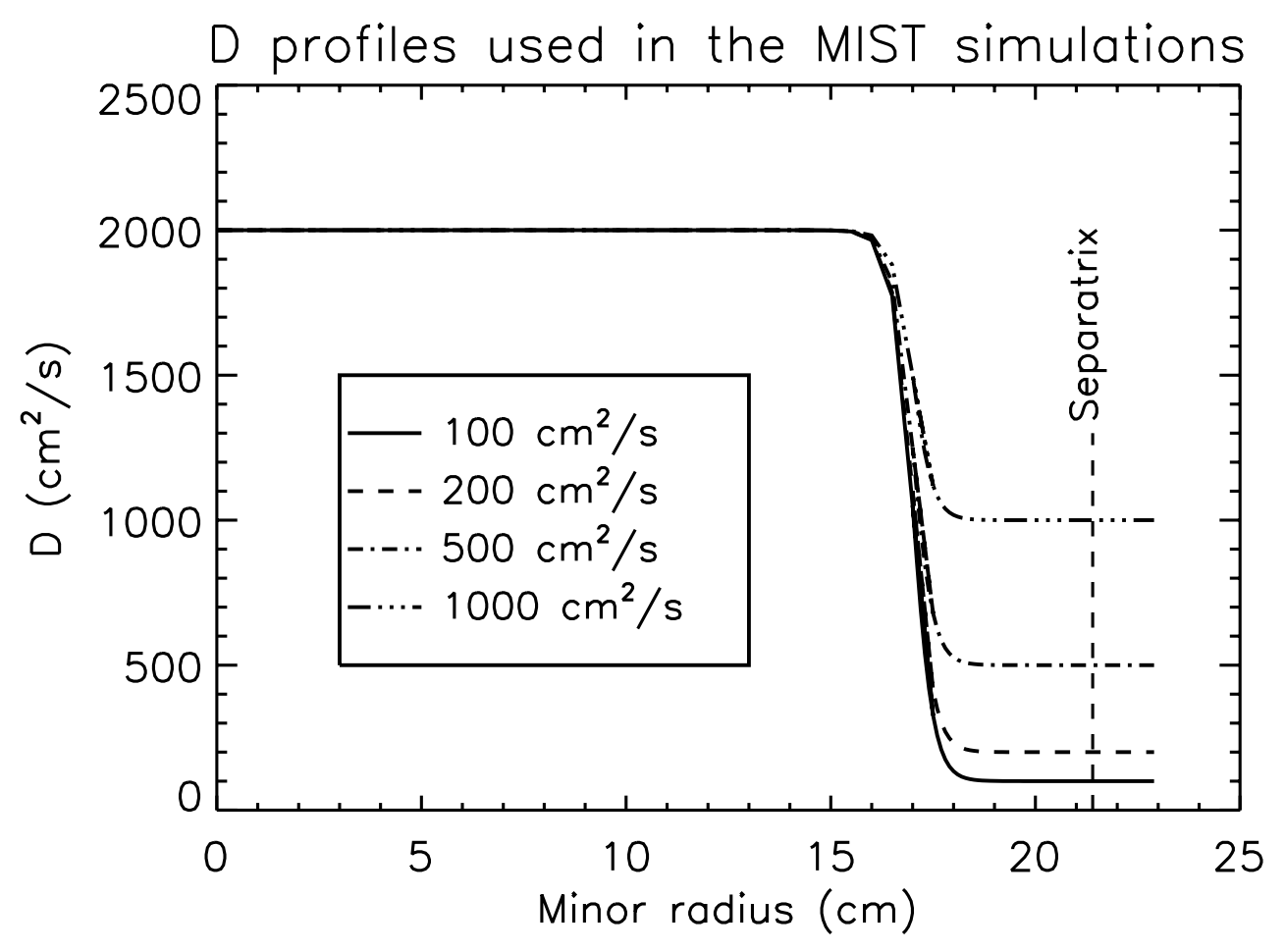

Figure 8: 


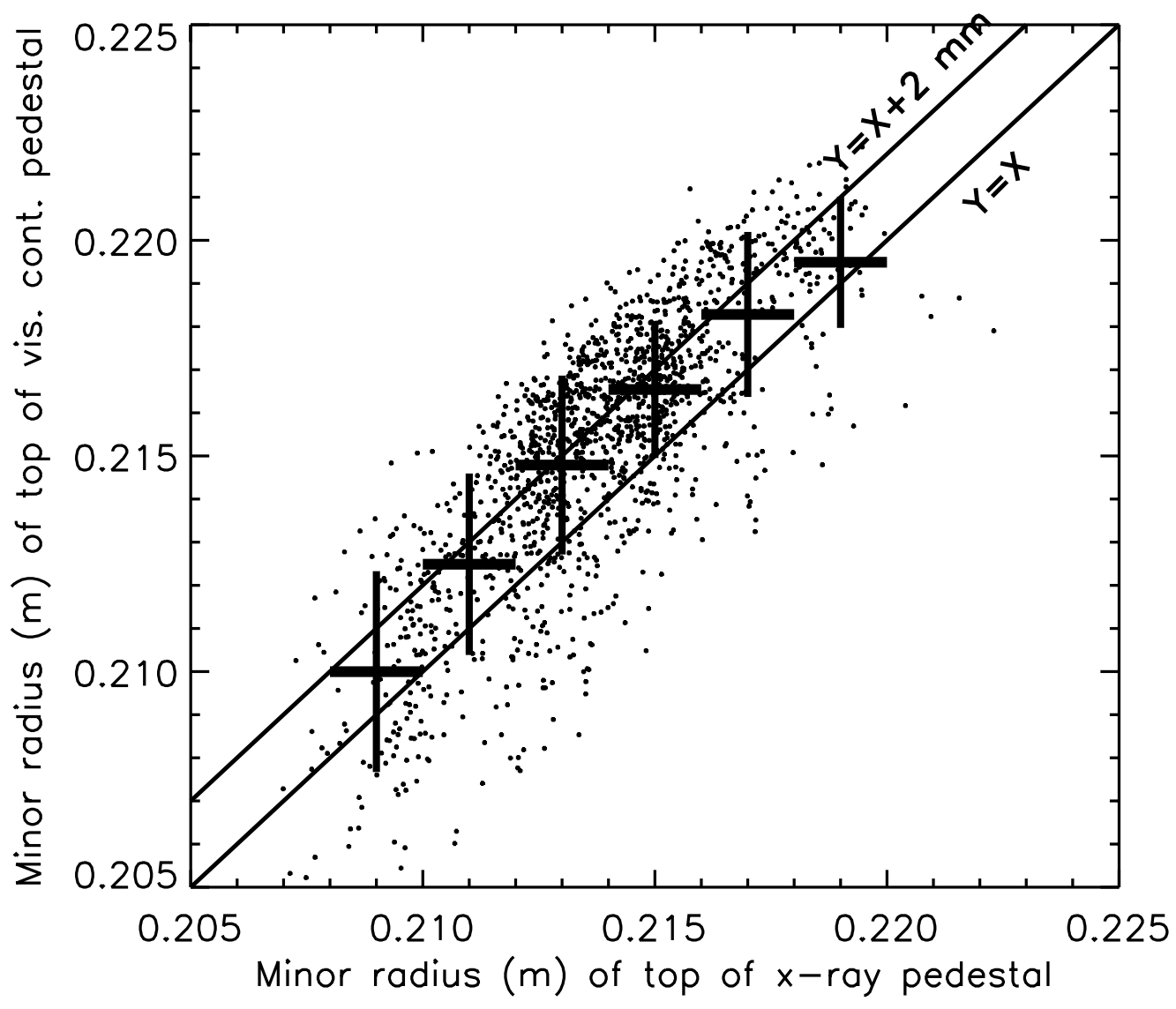

Figure 9: 


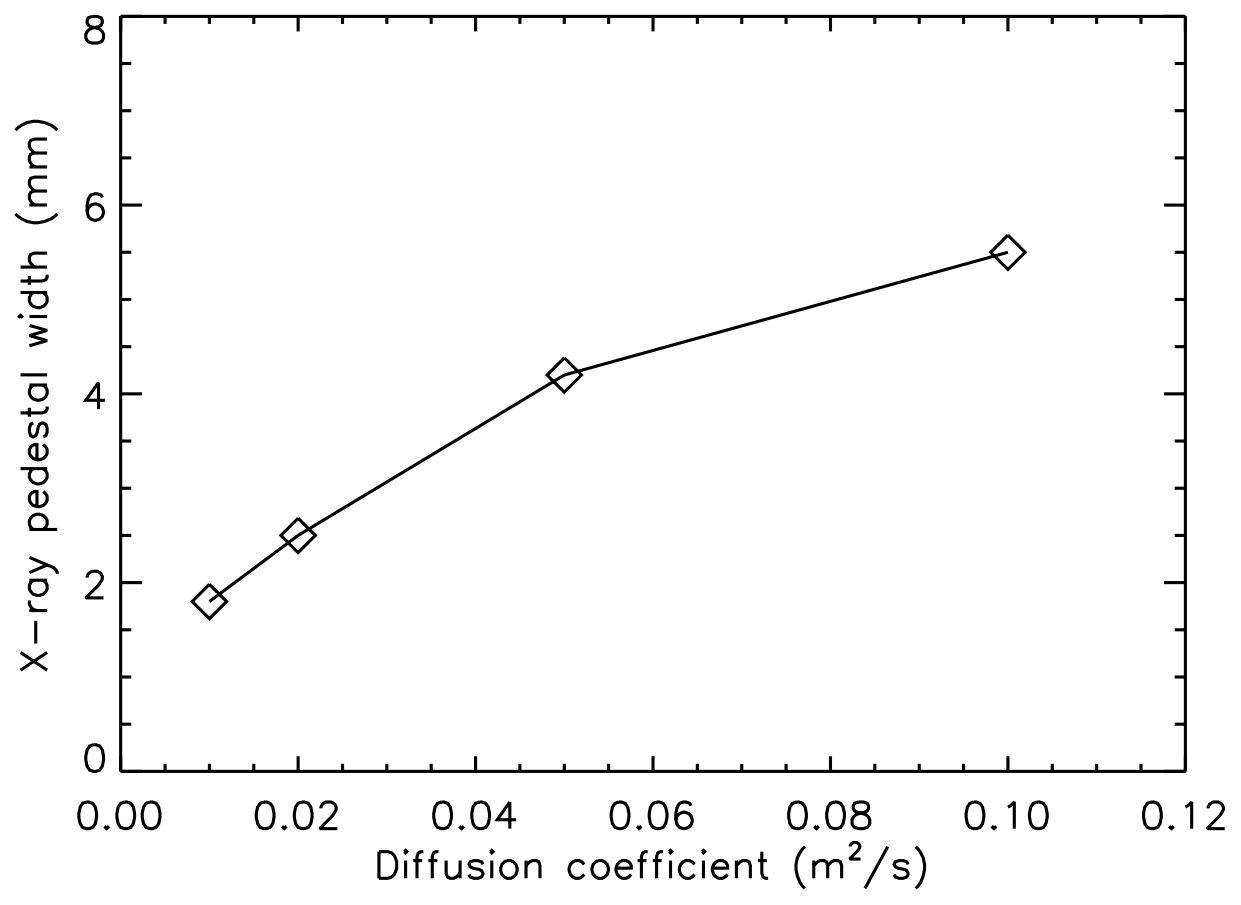

Figure 10: 


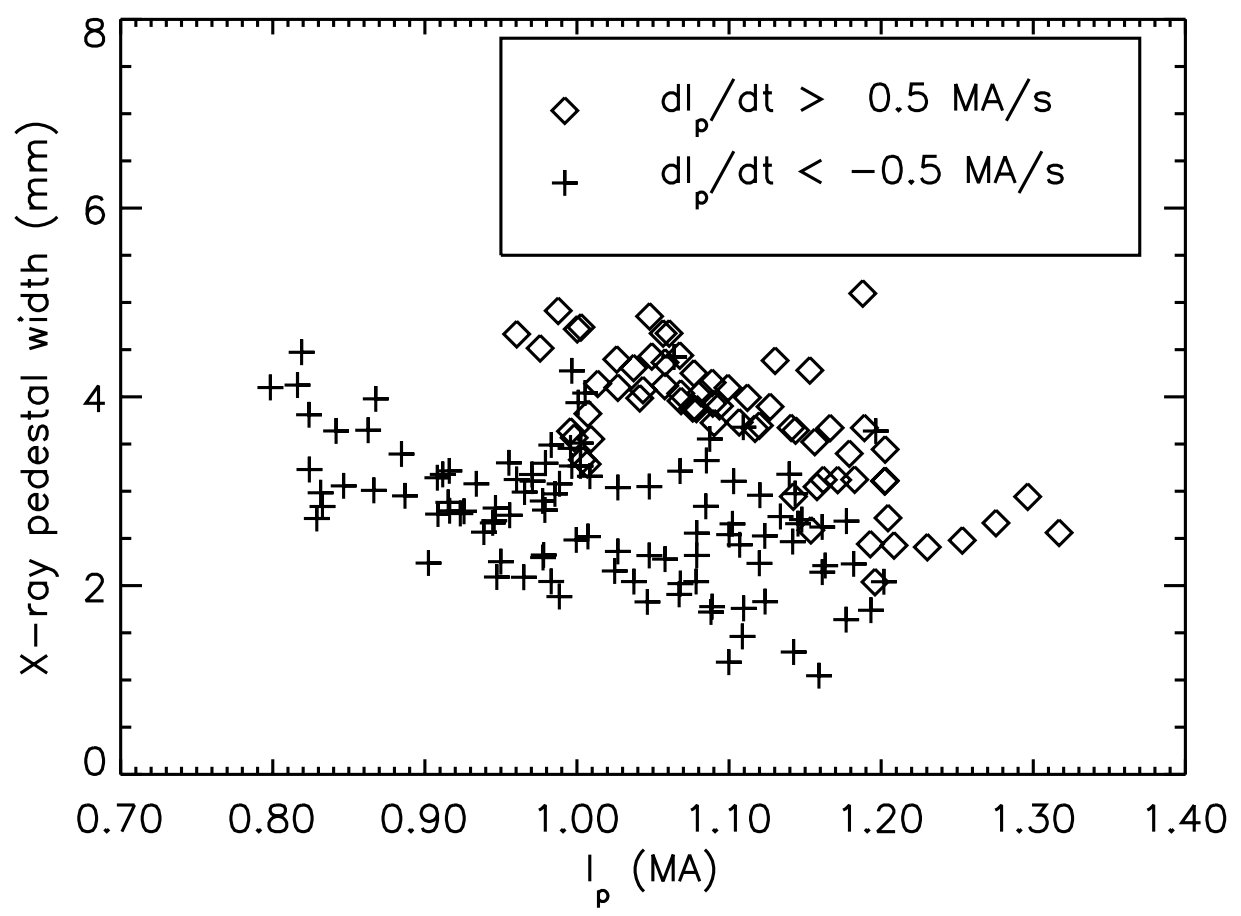

Figure 11: 